YITP-SB-01-25

LPT-Orsay-01-60

BNL-HET-01/24

\title{
Energy flow in QCD and event shape functions.
}

\author{
A.V. Belitsky ${ }^{a, b}$, G.P. Korchemsky ${ }^{b}$, G. Sterman ${ }^{a, c}$ \\ ${ }^{a}$ C.N. Yang Institute for Theoretical Physics \\ State University of New York at Stony Brook \\ NY 11794-3840, Stony Brook, USA \\ ${ }^{b}$ Laboratoire de Physique Théorique巾, Université de Paris XI \\ 91405 Orsay Cédex, France \\ ${ }^{c}$ Physics Department, Brookhaven National Laboratory \\ NY 11973-5000, Upton, USA
}

\begin{abstract}
Hadronization corrections to the thrust and related event shape distributions in the two-jet kinematical region of $\mathrm{e}^{+} \mathrm{e}^{-}$annihilation are summarized by nonperturbative shape functions. The moments of shape functions are given by universal matrix elements in QCD, which describe the energy flow in QCD final states. We show how the nonperturbative structure of these matrix elements may be inferred from resummed perturbation theory and Lorentz invariance. This analysis suggests the same functional forms for the shape functions as were found in phenomenological studies, and sheds light on the physical significance of the parameters that characterize these functions.
\end{abstract}

Keywords: QCD jets, hadronization, event shape distributions PACS numbers: 12.38.Cy, 12.38.Lg, 13.65.+i

\footnotetext{
${ }^{1}$ Unite Mixte de Recherche du CNRS (UMR 8627).
} 
1. Introduction. The predictive power of perturbative QCD is based on the factorization of long- and short-distance dynamics, made possible by their quantum-mechanical incoherence. The hard scattering of quarks and gluons can be computed in ordinary perturbation theory, while soft-scale physics is encoded into nonperturbative functions, which are fit to data, or evaluated using models. In a rather limited number of inclusive processes, which admit the operator product expansion (OPE), these functions are described by universal inclusive distributions, for example, leading-twist parton densities in deeply inelastic scattering. The latter can be defined in QCD in terms of correlators of quark and gluon fields on the light cone, and can, in principle, be computed using nonperturbative methods. On the other hand, there is a large variety of processes that are not completely inclusive, but are nevertheless infrared safe [1], and thus calculable in perturbation theory. Important examples are event shape distributions in $\mathrm{e}^{+} \mathrm{e}^{-}$final states [2]. Nonperturbative physics enters these cross sections as corrections, suppressed by powers of the large energy scale, $Q$.

Since event shape distributions are weighted, rather than fully inclusive, cross sections, their power corrections are not constrained by the OPE, and are generally less suppressed. For instance, the mean value of the thrust, $T$, acquires a nonperturbative correction $\langle t\rangle_{\mathrm{NP}} \sim \Lambda / Q$ with $t=1-T$, and $\Lambda$ a new QCD scale [3]. We may think of this correction as the first term in an expansion in $1 / Q$. The situation is even more complex for differential event shape distributions, such as $d \sigma / d t$, in the two-jet kinematic region, $t \rightarrow 0$. In this case, one has to deal with an infinite number of scales, $(d \sigma / d t)_{\mathrm{NP}} \sim \sum_{k} \Lambda_{k} /(t Q)^{k}$. The organization of such a series is equivalent to the introduction of a new nonperturbative distribution. These distributions are the shape functions [4, 5]. They provide a successful phenomenological description of differential event shapes, down to the two-jet limit, and over a wide interval of energies, as shown in Refs. [6, 7]. In this paper, we show how the functional form found in these phenomenological studies may be motivated from QCD.

A perturbative analysis of the event shape distributions in the two-jet region shows that the leading power corrections, $1 /(t Q)^{k}$, are associated with multiple soft, wide-angle gluon emission at the momentum scale $t Q[4,5]$. The influence of collinear splittings of quarks and gluons is suppressed by an extra power of $Q$. The leading behavior of the cross section is thus independent of the dynamics that produces the internal structure of jets, and is well described by a physical picture of the perturbative final state in which two fast moving back-to-back quarks propagate through a cloud of soft gluons, behaving as classical sources of color charge. This is equivalent to the eikonal approximation for the quark-antiquark pair.

In eikonal approximation, multiple soft-gluon emission from quarks is described by nonabelian phase operators $\Phi_{n_{R}}[\infty, y]$ and $\Phi_{n_{L}}^{\dagger}[\infty, y]$. Here $\Phi_{n}[\infty, y]=P \exp \left(i g \int_{0}^{\infty} d \sigma n^{\mu} A_{\mu}(\sigma n+y)\right)$ is a 
Wilson line, with its light-like direction $n_{\mu}$ defined by a quark momentum, $p_{1}^{\mu}=n_{L}^{\mu} \sqrt{Q^{2} / 2}$ or $p_{2}^{\mu}=n_{R}^{\mu} \sqrt{Q^{2} / 2}$, with $Q^{2}=\left(p_{1}+p_{2}\right)^{2}$ the total center-of-mass energy. In a frame where the two-jet axis points in the $z$-direction, we choose the four-velocities $n_{R}^{\mu}=\frac{1}{\sqrt{2}}(1, \mathbf{0}, 1)$ and $n_{L}^{\mu}=\frac{1}{\sqrt{2}}(1, \mathbf{0},-1)$, with $n_{L}^{2}=n_{R}^{2}=0$ and $n_{L} \cdot n_{R}=1$. Combining the eikonal phases of quark and antiquark, we obtain

$$
U(y ; A)=T\left\{\Phi_{n_{R}}[\infty, y] \Phi_{n_{L}}^{\dagger}[\infty, y]\right\}=T\left\{\Phi_{n_{R}}[\infty, y] \Phi_{n_{L}}[y, \infty]\right\}
$$

where we have used the unitarity of the phase operators. The symbol $T$ stands for time ordering of the gauge fields $A_{\mu}$ in the product. The explicit expression for $U$ can then be written as

$$
U(y ; A)=T P \exp \left(i g \int_{C_{\mathrm{e}^{+} \mathrm{e}^{-}}} d z^{\mu} A_{\mu}(y+z)\right),
$$

where $P$ orders the color indices of gluon fields along the integration path $C_{\mathrm{e}^{+} \mathrm{e}^{-}}: z^{\mu}(\tau)=$ $\tau n_{R}^{\mu} \theta(-\tau)+\tau n_{L}^{\mu} \theta(\tau)$ with $-\infty<\tau<\infty$.

A characteristic of weighted cross sections like the thrust and the heavy jet mass is that they distinguish between gluons moving into the right and left hemispheres, defined with respect to the plane orthogonal to the two-jet axis (in the overall center-of-mass frame). When the quarks are treated in the eikonal approximation, the shape function that describes energy flow for these final states is [由, 5]

$$
f\left(\varepsilon_{R}, \varepsilon_{L}\right)=\sum_{N}\left|\left\langle 0|U(0)| k_{1}, \ldots, k_{N}\right\rangle\right|^{2} \delta\left(\varepsilon_{R}-\sum_{i \in R} k_{i,+}\right) \delta\left(\varepsilon_{L}-\sum_{j \in L} k_{j,-}\right) .
$$

Here, the summation goes over soft particles in the final states, with momenta $k_{1}, \ldots, k_{N}$. The variables in the shape function, $\varepsilon_{R}$ and $\varepsilon_{L}$, are projections of the total momenta of soft particles flowing into the right and left hemispheres onto the corresponding jet directions,

$$
k_{+} \equiv k \cdot n_{R}, \quad k_{-} \equiv k \cdot n_{L}
$$

The operator $U(0)$ in Eq. (3) represents radiation from the quark-antiquark pair, in the eikonal approximation discussed above.

To derive a practical operator interpretation for the event shape function (3) in QCD, it is convenient to eliminate the summation over final states. To this end, we introduce an energy flow operator, $\mathcal{E}(\vec{n})$, which acts on asymptotic states by measuring the differential distribution of particles with energy $k^{0}$ moving in the solid angle $d \vec{n} \delta\left(\vec{n}^{2}-1\right)=d \cos \chi d \varphi$ [8, 9, 10]. We define this operator by its action on a final state consisting of $N$ particles with momenta $k_{1}, \ldots, k_{N}$, according to

$$
\mathcal{E}(\vec{n})\left|k_{1}, \ldots, k_{N}\right\rangle=\sum_{j=1}^{N} k_{j}^{0} \delta\left(\cos \theta_{j}-\cos \chi\right) \delta\left(\phi_{j}-\varphi\right)\left|k_{1}, \ldots, k_{N}\right\rangle,
$$




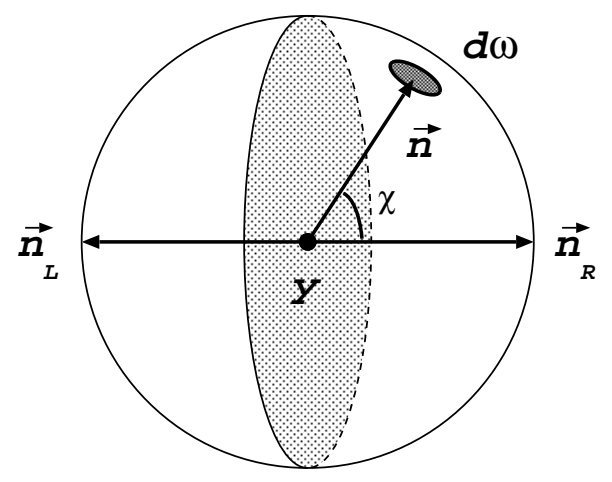

Figure 1: The pictorial definition of the energy flow operator $\mathcal{E}(\vec{n})$. The unit vectors $\vec{n}_{R}$ and $\vec{n}_{L}$ indicate the directions of the two outgoing jets. The shaded plane orthogonal to these unit vectors goes through the annihilation point, and separates the left and right hemispheres. The energy flux through the infinitesimal surface element $d \omega=d \vec{n} \delta\left(\vec{n}^{2}-1\right)$ is given by $\mathcal{E}(\vec{n}) d \omega$.

where spherical angles $\left(\theta_{j}, \phi_{j}\right)$ define the position of the unit vector $\vec{n}_{j}=\vec{k}_{j} /\left|\vec{k}_{j}\right|$ with respect to the jet axis $\vec{n}_{R}$, and $(\chi, \varphi)$ define those of $\vec{n}$. Introducing a light-like vector $n^{\mu}=(1, \vec{n})$, we can represent the total momentum flow through the right $\left(n^{3}>0\right)$ and left $\left(n^{3}<0\right)$ hemispheres of infinite radius in terms of

$$
\mathcal{P}_{R, L}^{\mu}=\int d \vec{n} n^{\mu} \theta\left( \pm n^{3}\right) \delta\left(\vec{n}^{2}-1\right) \mathcal{E}(\vec{n})
$$

so that

$$
\mathcal{P}_{R}^{\mu}\left|k_{1}, \ldots, k_{N}\right\rangle=\sum_{j \in R} k_{j}^{\mu}\left|k_{1}, \ldots, k_{N}\right\rangle
$$

and similarly for $\mathcal{P}_{L}^{\mu}$. Notice that the total momentum of the final state is given by the sum $\mathcal{P}^{\mu}=\int d \vec{x} \Theta^{0 \mu}(t, \vec{x})=\mathcal{P}_{R}^{\mu}+\mathcal{P}_{L}^{\mu}$, with $\Theta^{\mu \nu}$ the energy-momentum tensor.

Using these definitions, the shape function, Eq. (函), can be reexpressed as

$$
f\left(\varepsilon_{R}, \varepsilon_{L}\right)=\left\langle 0\left|U^{\dagger}(0) \delta\left(\varepsilon_{R}-\mathcal{E}_{R}\right) \delta\left(\varepsilon_{L}-\mathcal{E}_{L}\right) U(0)\right| 0\right\rangle
$$

where

$$
\mathcal{E}_{R, L}=\int d \vec{n} \delta\left(\vec{n}^{2}-1\right) w_{R, L}(\vec{n}) \mathcal{E}(\vec{n}), \quad w_{R, L}(\vec{n})=(1-|\cos \chi|) \theta( \pm \cos \chi)
$$

Defined in this way, the shape function becomes a new QCD distribution, which governs nonperturbative power corrections to a number of differential cross sections in the two-jet region.

A typical application of the shape function, which illustrates the factorization of soft gluon emission from perturbative dynamics, is to the distribution for the heavy jet mass. The heavy jet mass is defined by $\varrho=\left(1 / Q^{2}\right) \max \left(m_{R}^{2}, m_{L}^{2}\right)$, with $m_{R}\left(m_{L}\right)$ the invariant mass of the particles 
moving into the right (left) hemisphere. It may be written in the convolution form [5],

$$
\frac{1}{\sigma_{\text {tot }}} \frac{d \sigma_{\varrho}}{d \varrho}=Q f_{\varrho}(\varrho Q, \varrho Q) \mathcal{R}_{J}^{\mathrm{PT}}(0)+\int_{0}^{\varrho Q} d \varepsilon f_{\varrho}(\varepsilon, \varrho Q) \frac{d \sigma_{J}^{\mathrm{PT}}\left(\varrho-\frac{\varepsilon}{Q}\right)}{d \varrho}
$$

where $d \sigma_{J}^{\mathrm{PT}} / d \varrho$ is the resummed perturbative single-jet cross section [11], $\mathcal{R}_{J}^{\mathrm{PT}}$ is the corresponding 'radiator function', defined by $d \mathcal{R}_{J}^{\mathrm{PT}}(\varrho) / d \varrho=d \sigma_{J}^{\mathrm{PT}} / d \varrho$, and

$$
f_{\varrho}(\varepsilon, \varrho Q)=2 \int_{0}^{\varrho Q} d \varepsilon^{\prime} f\left(\varepsilon, \varepsilon^{\prime}\right) \mathcal{R}_{J}^{\mathrm{PT}}\left(\varrho-\frac{\varepsilon^{\prime}}{Q}\right) .
$$

The thrust and the $C$-parameter distributions are given by similar expressions [6], where $f_{\varrho}$ is replaced by a single-variable function, related to $f\left(\varepsilon_{R}, \varepsilon_{L}\right)$ by ${ }^{2}$

$$
f_{t}(\varepsilon)=\int_{0}^{\infty} d \varepsilon_{R} d \varepsilon_{L} f\left(\varepsilon_{R}, \varepsilon_{L}\right) \delta\left(\varepsilon-\varepsilon_{L}-\varepsilon_{R}\right)=\left\langle\delta\left(\varepsilon-\mathcal{E}_{R}-\mathcal{E}_{L}\right)\right\rangle .
$$

Corrections to Eq. (10) are suppressed by powers of $Q$, as discussed above.

In Ref. [6], it was found that a successful description of the differential distributions for thrust, heavy jet mass, and $C$-parameter over a wide range of energies is given by the single shape function,

$$
f\left(\varepsilon_{R}, \varepsilon_{L}\right)=\frac{\mathcal{N}(a, b)}{\Lambda^{2}}\left(\frac{\varepsilon_{R} \varepsilon_{L}}{\Lambda^{2}}\right)^{a-1} \exp \left(-\frac{\varepsilon_{R}^{2}+\varepsilon_{L}^{2}+2 b \varepsilon_{R} \varepsilon_{L}}{\Lambda^{2}}\right)
$$

when its parameters are chosen as: $a=2, b=-0.4$ and $\Lambda=0.55 \mathrm{GeV}$. The normalization factor $\mathcal{N}(a, b)$ is chosen so that $\int d \varepsilon_{R} d \varepsilon_{L} f\left(\varepsilon_{R}, \varepsilon_{L}\right)=1$. Recently, a thorough investigation of the differential distribution for thrust [7] reached similar conclusions in terms of the function $f_{t}$, Eq. (12). In the following, we shall argue that the functional form, Eq. (13), follows from a perturbative analysis of moments of the matrix element (8), supplemented by Lorentz invariance and standard treatment of integrals of the strong coupling over low momentum scales.

2. Energy correlators. In a manner analogous to the relation between the light-cone expansion and moments of deep-inelastic scattering structure functions, the moments of event shape functions give matrix elements of certain (in this case, nonlocal) operators in QCD. Specifically, from the definition, Eq. (8), the moments of $f\left(\varepsilon_{R}, \varepsilon_{L}\right)$ produce weighted Green functions of products of the operators $\mathcal{E}$, inserted into correlators of Wilson lines,

$$
\begin{array}{rl}
\int_{0}^{\infty} d \varepsilon_{R} d \varepsilon_{L} \varepsilon_{R}^{N} \varepsilon_{L}^{M} & f\left(\varepsilon_{R}, \varepsilon_{L}\right)=\left\langle\mathcal{E}_{R}^{N} \mathcal{E}_{L}^{M}\right\rangle \\
=\int \prod_{j=1}^{N+M} d \vec{n}_{j} \delta\left(\vec{n}_{j}^{2}-1\right) \prod_{n=1}^{N} w_{R}\left(\vec{n}_{n}\right) \prod_{m=N+1}^{M} w_{L}\left(\vec{n}_{m}\right) \mathcal{G}\left(\vec{n}_{1}, \ldots, \vec{n}_{N+M}\right),
\end{array}
$$

where

$$
\begin{aligned}
\mathcal{G}\left(\vec{n}_{1}, \ldots, \vec{n}_{N}\right) & =\left\langle\mathcal{E}\left(\vec{n}_{1}\right) \ldots \mathcal{E}\left(\vec{n}_{N}\right)\right\rangle \\
& \equiv \frac{1}{N_{c}}\left\langle 0\left|\operatorname{Tr}\left\{U^{\dagger}\left(0 ; A^{(-)}\right) \mathcal{E}\left(\vec{n}_{1}\right) \ldots \mathcal{E}\left(\vec{n}_{N}\right) U\left(0 ; A^{(+)}\right)\right\}\right| 0\right\rangle
\end{aligned}
$$

\footnotetext{
${ }^{2}$ In what follows we use a convention $\left\langle 0\left|U^{\dagger} \ldots U\right| 0\right\rangle \equiv\langle\ldots\rangle$.
} 
measures correlations between energy flows in the directions of specified unit vectors $\vec{n}_{j}$. Rather than study the shape function directly at first, we begin with these, even more general matrix elements, from which the shape function (8) may be derived [5].

We shall analyze below the energy correlators, $\mathcal{G}\left(\vec{n}_{1}, \ldots, \vec{n}_{N}\right)$, from a general point of view that has been widely applied to infrared safe quantities, using a variety of related prescriptions [3]. This may be summarized very briefly as follows. Starting with low-order diagrams for the quantity in question, we absorb higher-order corrections into the scale of the running coupling. In the resulting expression, the integral over the argument of the running coupling from zero up to a factorization scale, $\Lambda$, is replaced by a phenomenological parameter. Other, overall kinematic integrals, upon which the running coupling does not depend, determine properties like angular and rapidity dependence. We will show that this procedure, applied at order $\alpha_{s}$ and $\alpha_{s}^{2}$ to the energy correlators, is adequate to derive the functional form Eq. (13) for the corresponding shape functions, and to give physical interpretations to its dimensionless parameters $a$ and $b$.

The perturbative expansions of the correlators of Eq. (15) are illustrated diagrammatically by Fig. 2. As the figure shows, these correlation functions correspond to cut diagrams, rather than time-ordered Green functions, since the gauge fields entering the eikonal phases $U$ and $U^{\dagger}$ are time and anti-time ordered, respectively. To express this fact, we adopt the Keldysh notation, assigning "plus" and "minus" superscripts to the fields entering the amplitude $\left(A^{(+)}\right)$and its conjugated counterpart $\left(A^{(-)}\right)$. The propagator of the gauge fields $A^{( \pm)}$is defined as

$$
\int d^{4} x \mathrm{e}^{i q \cdot x}\left\langle 0\left|A_{\mu}^{(\alpha)}(x) A_{\nu}^{(\beta)}(0)\right| 0\right\rangle=-i \varrho_{\mu \nu}(q) D^{(\alpha \beta)}(q),
$$

with $\alpha, \beta= \pm$, and with the conventional free gluon polarization tensor in covariant gauge, $\varrho_{\mu \nu}(q)=g_{\mu \nu}-(1-\xi) q_{\mu} q_{\nu} / q^{2}$. In these terms, we have $D^{(++)}(q)=-\left[D^{(--)}(q)\right]^{\star}=\left(q^{2}+i 0\right)^{-1}$, $D^{(-+)}(q)=D^{(+-)}(-q)=-2 \pi i \theta\left(q^{0}\right) \delta\left(q^{2}\right)$. We choose below the Feynman gauge, $\xi=1$, for calculations (although our results are gauge invariant).

3. The single-gluon approximation. We begin our study of the properties of the correlation functions with the lowest-order perturbative expression for the single energy correlator, $\mathcal{G}(\vec{n})$. Using the definition (15) and expanding (2) in powers of the gauge coupling, we have

$$
\mathcal{G}(\vec{n})=g^{2} \int_{C_{\mathrm{e}^{+} \mathrm{e}^{-}}} d z_{1}^{\mu} \int_{C_{\mathrm{e}^{+} \mathrm{e}^{-}}} d z_{2}^{\nu}\left\langle 0\left|A_{\mu}^{(-)}\left(z_{1}\right) \mathcal{E}(\vec{n}) A_{\nu}^{(+)}\left(z_{2}\right)\right| 0\right\rangle+\mathcal{O}\left(g^{4}\right) .
$$

Going to the momentum representation, and performing the integration along the contour, we find

$$
\mathcal{G}(\vec{n})=\frac{C_{F} \alpha_{s}}{2 \pi^{2}} \int \frac{d k_{+}}{k_{+}} \int \frac{d \boldsymbol{k}^{2}}{\boldsymbol{k}^{2}} \int d \phi_{k} k_{0} \delta\left(\cos \theta_{k}-\cos \chi\right) \delta\left(\phi_{k}-\varphi\right) .
$$

In this formula, we recognize the lowest-order bremsstrahlung spectrum of soft gluons, weighted by an additional energy flow factor, according to Eq. (5). Using the definitions of the light-cone 


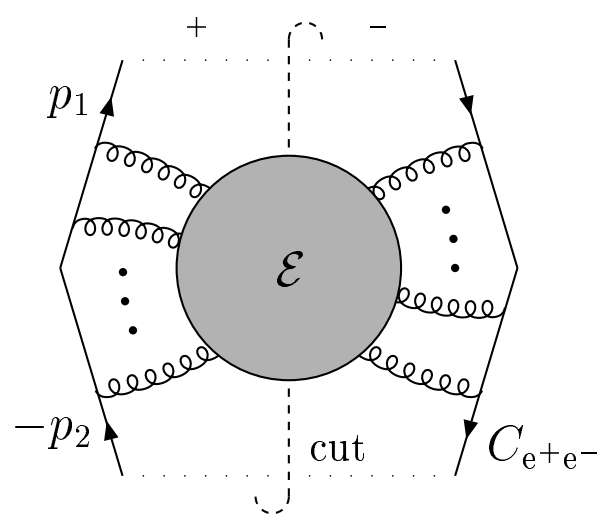

Figure 2: Diagrammatic representation of the correlation function (15). Here the dashed line represents the unitary cut and the final states are weighted with the product of factors $\mathcal{E}\left(\vec{n}_{1}\right) \ldots \mathcal{E}\left(\vec{n}_{N}\right)$ defined in Eq. (5).

variables $k_{ \pm}=\frac{1}{\sqrt{2}}\left(k_{0} \pm k_{3}\right)$, along with $k_{0}=\sqrt{\boldsymbol{k}^{2}} \sin ^{-1} \theta_{k}, k_{3}=\sqrt{\boldsymbol{k}^{2}} \cot \theta_{k}$, we find $d k_{+} / k_{+}=$ $d \cos \theta_{k} / \sin ^{2} \theta_{k}$. The integration over $k_{+}$then yields the result

$$
\mathcal{G}(\vec{n})=\frac{1}{2 \pi} \frac{1}{\sin ^{3} \chi} \int d \boldsymbol{k}^{2} \sqrt{\boldsymbol{k}^{2}} \rho_{\mathrm{PT}}\left(\boldsymbol{k}^{2}\right),
$$

where we have introduced a resummed perturbative density, $\rho_{\mathrm{PT}}\left(\boldsymbol{k}^{2}\right) \equiv \frac{C_{F}}{\pi} \frac{\alpha_{s}\left(\boldsymbol{k}^{2}\right)}{\boldsymbol{k}^{2}}$ [3], which measures the number of particles produced per unit interval in rapidity and transverse momentum. The expression (19) exhibits a third-order pole as $\chi \rightarrow 0$ or $\chi \rightarrow \pi$. In both cases, a gluon propagates in the final state close to the direction of an outgoing jet, and the resulting singularities are of collinear origin.

The simplicity of the $\chi$-dependence in Eq. (19) reflects the underlying boost-invariance of the nonabelian phase operators $U$. All dynamical information is contained in a boost-invariant particle density, independent of the rapidity,

$$
\eta \equiv \frac{1}{2} \ln \frac{k_{+}}{k_{-}}=-\ln \tan \frac{\chi}{2} .
$$

The resummation incorporated into the running coupling in the step from Eq. (18) to Eq. (19) is the only choice consistent with the boost properties of the correlators. This resummation, which summarizes higher-order scale-fixing corrections, $\alpha_{s}\left(\beta_{0} \alpha_{s}\right)^{N}$, is normally justified diagrammatically, and is used as a starting point for infrared renormalon-inspired arguments [3]. We shall not employ the form of the perturbative running coupling, however, nor will we make a specific ansatz for a nonperturbative generalization of the integrals in Eq. (19]). Following Refs. [3], however, we replace the resummed perturbative density by a phenomenological one, $\rho_{\mathrm{NP}}\left(\boldsymbol{k}^{2}\right)$, below some cutoff, $\Lambda^{2}$, in the $\boldsymbol{k}^{2}$ integral. Whatever the choice of $\rho_{\mathrm{NP}}$, it should, like its perturbative counterpart, be boost invariant. For any such choice of the nonperturbative density, the single-particle 
energy correlator, $\mathcal{G}(\vec{n})$, defines a leading-power correction to the energy-energy correlation in $\mathrm{e}^{+} \mathrm{e}^{-}$-annihilation [12],

$$
\langle\operatorname{EEC}(\chi)\rangle_{\mathrm{NP}}=\frac{1}{Q} \mathcal{G}(\vec{n}),
$$

with the rapidity dependence specified by Eqs. (19) and (20), and any choice also produces a uniform particle number distribution, $d^{2} N / d \eta d \boldsymbol{k}^{2}=\rho\left(\boldsymbol{k}^{2}\right)$, in terms of the rapidity.

We have thus, starting with the energy correlators, arrived at a "tube" model, as reviewed in Ref. [13, in which particles are produced with a constant, nonperturbative density at all rapidities. The perturbative calculation therefore suggests a realistic dependence on the rapidity, in terms of an integral of the density $\rho$ over nonperturbative scales. This integral, of course, is to be replaced by a set of phenomenological parameters. We now use this approach in the computation of multiple energy correlators. Perhaps surprisingly, the lowest-order model offers insights here as well.

To the lowest order in perturbation theory, the multiple energy flow correlator $\mathcal{G}\left(\vec{n}_{1}, \ldots, \vec{n}_{j}\right)$ is given by

$$
\mathcal{G}\left(\vec{n}_{1}, \ldots, \vec{n}_{j}\right)=g^{2} \int_{C_{\mathrm{e}^{+} \mathrm{e}^{-}}} d z_{1}^{\mu} \int_{C_{\mathrm{e}^{+} \mathrm{e}^{-}}} d z_{2}^{\nu}\left\langle 0\left|A_{\mu}^{(-)}\left(z_{1}\right) \mathcal{E}\left(\vec{n}_{1}\right) \ldots \mathcal{E}\left(\vec{n}_{j}\right) A_{\nu}^{(+)}\left(z_{2}\right)\right| 0\right\rangle+\mathcal{O}\left(g^{4}\right)
$$

whose evaluation leads to

$$
\mathcal{G}\left(\vec{n}_{1}, \ldots, \vec{n}_{j}\right)=\frac{1}{2 \pi} \frac{1}{\sin ^{j+2} \chi_{1}} \prod_{i=2}^{j} \delta\left(\cos \chi_{1}-\cos \chi_{i}\right) \delta\left(\varphi_{1}-\varphi_{i}\right) \int d \boldsymbol{k}^{2}\left(\sqrt{\boldsymbol{k}^{2}}\right)^{j} \rho_{\mathrm{PT}}\left(\boldsymbol{k}^{2}\right),
$$

where $\left(\chi_{i}, \varphi_{i}\right)$ are the spherical coordinates of the unit vector $\vec{n}_{i}$. Notice that this correlator vanishes unless all vectors are aligned. Higher order corrections, discussed below, will relax this condition.

Starting from Eq. (23), we easily calculate the total flow into the right or left hemispheres. According to (14), it is given by

$$
\left\langle\mathcal{E}_{L}^{N}\right\rangle=\left\langle\mathcal{E}_{R}^{N}\right\rangle=\frac{2}{N} \int_{0}^{\Lambda^{2}} d \boldsymbol{k}^{2}\left(\sqrt{\boldsymbol{k}^{2}}\right)^{N} \rho_{\mathrm{PT}}\left(\boldsymbol{k}^{2}\right)=2 C_{F} \frac{\alpha_{s}}{\pi} \frac{\Lambda^{N}}{N^{2}},
$$

where in the last relation we give the result for fixed coupling. The vanishing of inter-hemisphere correlations,

$$
\left\langle\mathcal{E}_{R}^{N} \mathcal{E}_{L}^{M}\right\rangle=0+\mathcal{O}\left(\alpha_{s}^{2}\right)
$$

implies that the shape function, $f\left(\varepsilon_{R}, \varepsilon_{L}\right)$, is factorized into the product of two functions, each depending on a single energy variable

$$
f_{\text {one-gluon }}\left(\varepsilon_{R}, \varepsilon_{L}\right)=f\left(\varepsilon_{R}\right) f\left(\varepsilon_{L}\right)
$$


with the function defined as $f\left(\varepsilon_{i}\right)=\left\langle\delta\left(\varepsilon_{i}-\mathcal{E}_{i}\right)\right\rangle$ for $i=R, L$. Nonfactorizable corrections to Eq. (26) begin with $\alpha_{s}^{2}$-contributions to multiple energy flow correlators $\mathcal{G}\left(\vec{n}_{1}, \ldots, \vec{n}_{j}\right)$ associated with final states with two real gluons. Phenomenological analyses [6, [7] have shown that power corrections to certain event shapes, in particular the heavy-jet mass, are very sensitive to these corrections.

4. Order $\alpha_{s}^{2}$ and correlations between hemispheres. To study correlations between hemispheres at lowest order, $\alpha_{s}^{2}$, in $\mathcal{G}\left(\vec{n}_{1}, \vec{n}_{2}\right)$, we restrict ourselves to Feynman diagrams in Fig. 2 involving two-gluon cuts. According to the color structure, one can separate abelian and nonabelian contributions, $\mathcal{G}=\mathcal{G}_{A}+\mathcal{G}_{N A}$.

The abelian part, $\mathcal{G}_{A}\left(\vec{n}_{1}, \vec{n}_{2}\right)$, arises from QED-like diagrams. Due to the exponentiation properties of eikonal lines [14], their contribution is factorized into the product of independent single-gluon emissions,

$$
\mathcal{G}_{A}\left(\vec{n}_{1}, \vec{n}_{2}\right)=\frac{\alpha_{s}^{2}}{4 \pi^{4}} \frac{C_{F}^{2}}{\sin ^{3} \chi_{1} \sin ^{3} \chi_{2}} \int_{0}^{\Lambda^{2}} \frac{d \boldsymbol{k}_{1}^{2}}{\sqrt{\boldsymbol{k}_{1}^{2}}} \frac{d \boldsymbol{k}_{2}^{2}}{\sqrt{\boldsymbol{k}_{2}^{2}}}=\mathcal{G}\left(\vec{n}_{1}\right) \mathcal{G}\left(\vec{n}_{2}\right),
$$

where $\mathcal{G}(\vec{n})$ is given by Eq. (18) and where we impose a cutoff, $\Lambda$, on gluon transverse momenta, as above. The overall factor $\left(\sin \chi_{1} \sin \chi_{2}\right)^{-3}$ corresponds to two independent collinear enhancements, when either gluon is emitted nearly parallel to one of the eikonal directions. For now, we study $\mathcal{G}_{A}\left(\vec{n}_{1}, \vec{n}_{2}\right)$ at fixed $\alpha_{s}$. We will comment afterward on the role of the infrared behavior of the running coupling.

The nonabelian part, $\mathcal{G}_{N A}\left(\vec{n}_{1}, \vec{n}_{2}\right)$, receives contributions from the crossed ladder, self-energy and triple-gluon vertex diagrams (see, e.g., Fig. 1 in Ref. 15]). The total expression is a somewhat complicated function depending on the spherical angles $\left(\chi_{1}, \varphi_{1}\right)$ and $\left(\chi_{2}, \varphi_{2}\right)$. Closer examination reveals, however, that $\mathcal{G}_{N A}\left(\vec{n}_{1}, \vec{n}_{2}\right)$ depends, up to a prefactor, only on two specific combinations of these angles

$$
\mathcal{G}_{N A}\left(\vec{n}_{1}, \vec{n}_{2}\right)=\frac{\alpha_{s}^{2}}{\pi^{2}} \frac{C_{F} C_{A}}{\sin ^{3} \chi_{1} \sin ^{3} \chi_{2}} \Lambda^{2} \mathcal{F}\left(\cosh \left(\eta_{2}-\eta_{1}\right), \cos \left(\varphi_{2}-\varphi_{1}\right)\right)
$$

with

$$
\cosh \left(\eta_{2}-\eta_{1}\right)=\frac{1}{2} \tan \frac{\chi_{1}}{2} \cot \frac{\chi_{2}}{2}+\frac{1}{2} \tan \frac{\chi_{2}}{2} \cot \frac{\chi_{1}}{2}
$$

where the rapidity $\eta_{i}$ is defined in Eq. (20). This property is a consequence of boost invariance along the $z$-direction, and rotational invariance around it. The explicit expression for the dimensionless function $\mathcal{F}$ is rather cumbersome, and in fact we do not need it for our purposes. Indeed, the correlator $\mathcal{G}$ enters into the expression for the moments of the shape function (14) under the integral over azimuthal angles $\varphi_{i}$. Since the weights $w_{R, L}$ (9) do not depend on the $\varphi_{i}$, we may integrate over the azimuthal angles, to arrive at

$$
\int_{0}^{2 \pi} d \varphi_{1} \int_{0}^{2 \pi} d \varphi_{2} \mathcal{G}_{N A}\left(\vec{n}_{1}, \vec{n}_{2}\right)=\frac{\alpha_{s}^{2}}{\pi^{2}} \frac{C_{F} C_{A}}{\sin ^{3} \chi_{1} \sin ^{3} \chi_{2}} \Lambda^{2} \overline{\mathcal{F}}\left(\left|\eta_{2}-\eta_{1}\right|\right),
$$


where

$$
\overline{\mathcal{F}}(\eta)=\frac{1}{(2 \Lambda)^{2}} \int_{0}^{\Lambda^{2}} \frac{d \boldsymbol{k}_{1}^{2}}{\sqrt{\boldsymbol{k}_{1}^{2}}} \frac{d \boldsymbol{k}_{2}^{2}}{\sqrt{\boldsymbol{k}_{2}^{2}}} \frac{1+D\left(\boldsymbol{k}_{1}^{2}, \boldsymbol{k}_{2}^{2}\right)}{D^{2}\left(\boldsymbol{k}_{1}^{2}, \boldsymbol{k}_{2}^{2}\right)}\left\{D\left(\boldsymbol{k}_{1}^{2}, \boldsymbol{k}_{2}^{2}\right)(\operatorname{coth} \eta-1)-\frac{\sqrt{\boldsymbol{k}_{1}^{2} \boldsymbol{k}_{2}^{2}}}{\boldsymbol{k}_{1}^{2}+\boldsymbol{k}_{2}^{2}} \frac{1}{\sinh \eta}\right\}
$$

with the function $D$ defined as

$$
D\left(\boldsymbol{k}_{1}^{2}, \boldsymbol{k}_{2}^{2}\right)=1+2 \frac{\sqrt{\boldsymbol{k}_{1}^{2} \boldsymbol{k}_{2}^{2}}}{\boldsymbol{k}_{1}^{2}+\boldsymbol{k}_{2}^{2}} \cosh \eta
$$

Integrating over transverse momenta, we find

$$
\begin{gathered}
\overline{\mathcal{F}}(\eta)=(\operatorname{coth} \eta-1)(2+\eta \cosh \eta \operatorname{coth} \eta)+\frac{\cosh \eta}{2 \sinh ^{4} \eta}\left(2 \eta \cosh ^{2} \eta-3 \eta+\sinh \eta\right) \\
-\left(\operatorname{coth} \eta-1+\frac{1}{\sinh \eta}\right) \ln (2+2 \cosh \eta)
\end{gathered}
$$

The function $\overline{\mathcal{F}}$ depends only on the relative rapidity, $\eta=\left|\eta_{1}-\eta_{2}\right|$, of the emitted gluons. We find that at large $\eta$ it decreases exponentially

$$
\overline{\mathcal{F}}(\eta)=2 \eta \mathrm{e}^{-3 \eta}+\mathcal{O}\left(\mathrm{e}^{-4 \eta}\right)
$$

while at small $\eta$ it has a pole,

$$
\overline{\mathcal{F}}(\eta)=\frac{1}{\eta}\left\{\frac{49}{12}-4 \ln 2\right\}+\mathcal{O}\left(\eta^{0}\right) .
$$

These properties may be understood as follows.

When $\chi_{k} \rightarrow 0, \pi$, the $k$-th gluon is emitted collinear to one of the eikonal directions. At first sight, the nonabelian contribution, Eq. (28) has the same double collinear enhancement as the abelian term, (27) from the factor $\left(\sin \chi_{1} \sin \chi_{2}\right)^{-3}$. The large- $\eta$ behavior $\exp (-3 \eta)$ in $\overline{\mathcal{F}}(\eta)$, however, prevents enhancements when the directions of the gluons are widely separated in rapidity. The nonabelian term therefore has at most a single collinear enhancement from configurations where both gluons approach the direction of one of the eikonal lines. This result is consistent with the exponentiation of collinear logarithms in inclusive cross sections, which requires that the double-logarithmic collinear enhancement at two loops be given by the square of the enhancement at one loop.

At small rapidity intervals, $\left|\eta_{2}-\eta_{1}\right| \rightarrow 0$, at fixed $\eta_{k} \neq 0, \pi$, the gluons propagate in the same direction. This gives rise to another collinear pole, $1 / \eta$, from (35), which is compensated by a virtual $\mathcal{O}\left(\alpha_{s}\right)$ correction to single gluon emission. Once combined, they define a distribution regularized via a 'plus'-prescription, and give no new collinear enhancement to any infrared safe cross section. 
Let us examine the contribution of the correlator $\mathcal{G}\left(\vec{n}_{1}, \vec{n}_{2}\right)$ to the total energy flow into the left and/or right hemispheres, Eqs. (24) and (25). We find that (24) receives an $\mathcal{O}\left(\alpha_{s}^{2}\right)$ correction, while (25) develops a non-zero value. The leading order $\alpha_{s}^{2}$-contribution to the correlator $\left\langle\mathcal{E}_{R}^{M} \mathcal{E}_{L}^{N}\right\rangle$ is naturally decomposed into an abelian $\left(\sim C_{F}^{2}\right)$ and nonabelian $\left(\sim C_{F} C_{A}\right)$ part. Due to the factorization property, (27), the former is reduced to the product of two factors, $\left\langle\mathcal{E}_{R}^{M}\right\rangle\left\langle\mathcal{E}_{L}^{N}\right\rangle$, each given by the lowest order expression (24). The nonabelian piece defines the irreducible part of the correlator $\left\langle\mathcal{E}_{R} \mathcal{E}_{L}\right\rangle$,

$$
\left\langle\left\langle\mathcal{E}_{R} \mathcal{E}_{L}\right\rangle\right\rangle=\left\langle\mathcal{E}_{R} \mathcal{E}_{L}\right\rangle-\left\langle\mathcal{E}_{R}\right\rangle\left\langle\mathcal{E}_{L}\right\rangle
$$

It is given by the real-gluon contribution to $\mathcal{G}_{N A}\left(\vec{n}_{1}, \vec{n}_{2}\right)$ alone, evaluated in Eq. (30). Because $\vec{n}_{1}$ and $\vec{n}_{2}$ belong to different hemispheres, the virtual gluon contribution is non-vanishing only for $\chi_{1}=\chi_{2}=\pi / 2$. This corresponds to a single point in the phase space, $0 \leq \chi_{1} \leq \pi / 2$ and $\pi / 2 \leq \chi_{2} \leq \pi$, and vanishes upon integration over $\chi_{1,2}$. Substituting the result of the perturbative computation of Eqs. (30) and (33), one finds after some algebra

$$
\left\langle\left\langle\mathcal{E}_{R} \mathcal{E}_{L}\right\rangle\right\rangle=\frac{\alpha_{s}^{2}}{\pi^{2}} C_{F} C_{A} \Lambda^{2} \int_{0}^{\infty} d \eta \eta \mathrm{e}^{-\eta} \overline{\mathcal{F}}(\eta) \approx \frac{\alpha_{s}^{2}}{\pi^{2}} C_{F} C_{A} \Lambda^{2} \cdot 0.5314
$$

This correlator describes cross-talk between the two hemispheres and it is positive. As we will see below, this has direct consequences for the differential event shape distributions, confirmed by phenomenological analysis [6].

So far, we have kept the couplings fixed in our investigation of $\left\langle\left\langle\mathcal{E}_{R} \mathcal{E}_{L}\right\rangle\right\rangle$. The inclusion of higher order logarithmic corrections, of course, requires a resummation, and we certainly do not regard the numerical value of the correlation in (37) as a prediction. The positive sign of $\left\langle\left\langle\mathcal{E}_{R} \mathcal{E}_{L}\right\rangle\right\rangle$, however, is stable under any resummation that incorporates long-distance effects through the running of the couplings with transverse momenta, and even as functions of the relative rapidity $\eta$. This follows from the form of integrand of Eq. (33), which is positive-definite for any fixed values of the transverse momenta and $\eta$. Similarly, the explicit $\chi_{i}$-dependence in (28) is a direct consequence of boost invariance, and holds both perturbatively and nonperturbatively.

5. From the correlators to the shape function. We are now ready to apply the expressions that we have obtained for the energy flow correlators to reconstruct the shape function $f\left(\varepsilon_{R}, \varepsilon_{L}\right)$. We start with the single-gluon approximation, in which the factorization property (26) holds, and for which the correlations of energy flow are given by Eqs. (24) and (25). It follows from (26) that

$$
f\left(\varepsilon_{R}\right)=\left\langle\delta\left(\varepsilon_{R}-\mathcal{E}_{R}\right)\right\rangle=\int_{-\infty}^{\infty} \frac{d \lambda}{2 \pi} \mathrm{e}^{i \lambda \varepsilon_{R}}\left\langle\exp \left(-i \lambda \mathcal{E}_{R}\right)\right\rangle
$$

and similarly for $f\left(\varepsilon_{L}\right)$. To calculate the expectation value entering this expression, we use the cumulant expansion,

$$
\left\langle\mathrm{e}^{X}\right\rangle=\exp \sum_{j=1}^{\infty} \frac{1}{j !}\left\langle\left\langle X^{j}\right\rangle\right\rangle
$$


where the lowest cumulants are, $\langle\langle 1\rangle\rangle \equiv\langle 1\rangle,\langle\langle 12\rangle\rangle \equiv\langle 12\rangle-\langle 1\rangle\langle 2\rangle,\langle\langle 123\rangle\rangle \equiv\langle 123\rangle-\langle 1\rangle\langle 23\rangle-$ $\langle 12\rangle\langle 3\rangle-\langle 13\rangle\langle 2\rangle+2\langle 1\rangle\langle 2\rangle\langle 3\rangle$, and analogously for higher cumulants (see, for example [16]). In introducing the cumulant expansion, we note the close resemblance to the classic analysis of particle multiplicity, [17.

The shape function $f\left(\varepsilon_{R}\right)$ is found by substituting $X=-i \lambda \mathcal{E}_{R}$ in (39). In the one-gluon approximation, $\left\langle\left\langle\mathcal{E}^{N}\right\rangle\right\rangle=\left\langle\mathcal{E}^{N}\right\rangle$. Then, using the correlators (24), we easily resum the series in (38) and obtain

$$
f\left(\varepsilon_{R}\right)=\int_{-\infty}^{\infty} \frac{d \lambda}{2 \pi} \exp \left\{i \lambda \varepsilon_{R}+\int_{0}^{\Lambda^{2}} d \boldsymbol{k}^{2} \rho_{\mathrm{PT}}\left(\boldsymbol{k}^{2}\right) \int_{0}^{\infty} d \eta\left(\mathrm{e}^{-i \lambda \sqrt{\boldsymbol{k}^{2}} \mathrm{e}^{-\eta}}-1\right)\right\}
$$

and analogously for $f\left(\varepsilon_{L}\right)$. It is straightforward to verify that $f\left(\varepsilon_{R, L}\right)$ is a positive definite distribution. The function we have just calculated describes a resummed single-gluon contribution to the shape function. Its lowest-order expansion in powers of $\alpha_{s}$ reproduces a familiar $1 / \varepsilon_{R}$ energy distribution,

$$
f\left(\varepsilon_{R}\right)=\frac{1}{\varepsilon_{R}} \theta\left(\Lambda-\varepsilon_{R}\right) \int_{\varepsilon_{R}^{2}}^{\Lambda^{2}} d \boldsymbol{k}^{2} \rho_{\mathrm{PT}}\left(\boldsymbol{k}^{2}\right)+\mathcal{O}\left(\alpha_{s}^{2}\right) .
$$

At small $\varepsilon_{R}$, higher-order corrections become important, and the asymptotic behavior of the resummed expression is drastically changed. To see this, we notice that the $\varepsilon_{R} \rightarrow 0$ behavior of (40) is determined by the asymptotic behavior of the integrand as $\lambda \rightarrow \infty$. In this limit, using $\left.\int_{0}^{\infty} d \eta\left\{\exp \left(-i \lambda \sqrt{\boldsymbol{k}^{2}} \mathrm{e}^{-\eta}\right)-1\right\}\right|_{\lambda \rightarrow \infty} \propto-\ln \lambda$, we find

$$
f\left(\varepsilon_{R}\right) \stackrel{\varepsilon_{R} \rightarrow 0}{\sim} \varepsilon_{R}^{a_{\mathrm{PT}}-1}, \quad a_{\mathrm{PT}}=\int_{0}^{\Lambda^{2}} d \boldsymbol{k}^{2} \rho_{\mathrm{PT}}\left(\boldsymbol{k}^{2}\right) .
$$

Here $a_{\mathrm{PT}}$ has the interpretation of the number of particles per unit rapidity interval. Perturbation theory thus suggests that $f$ vanishes as a power of the energy, although it cannot reliably predict the absolute value of the exponent $a_{\mathrm{PT}}$. In particular, $a_{\mathrm{PT}}$ suffers from infrared renormalon ambiguities related to the divergence of the coupling constant at small values of $\boldsymbol{k}^{2}$. One can estimate $a_{\mathrm{NP}} \approx 1-3$, replacing $\rho_{\mathrm{PT}}$ by a phenomenologically motivated $\rho_{\mathrm{NP}}[13]$.

Now let us consider slightly larger values of $\varepsilon_{R}$, for which Eq. (40) is dominated by finite values of $\lambda$, that is, values such that $\lambda \sqrt{\kappa^{2}}$ is a number of order unity for all $\kappa^{2} \leq \Lambda^{2}$. In this region, we may expand the exponential, $\exp \left(-i \lambda \sqrt{\boldsymbol{k}^{2}} \mathrm{e}^{-\eta}\right)$ as a power series in $\lambda$. If we keep only the linear term in $\lambda$, we get

$$
f^{(0)}\left(\varepsilon_{R}\right) \propto \delta\left(\varepsilon_{R}-\left\langle\mathcal{E}_{R}\right\rangle\right)
$$

where the superscript refers to the linear approximation used in (40). This model for the shape function is equivalent to a "shift" [3] of the perturbative distribution in Eq. (10).

Clearly, a simple shift cannot readily be combined with the low- $\varepsilon_{R}$ behavior of Eq. (42). On the other hand, the shift results from keeping only the lowest order in $\lambda$ in our expansion of the 
exponent in Eq. (40). The inclusion of the next order, $\lambda^{2}$, term amounts to a smearing of the delta-function into a Gaussian form

$$
f^{(1)}\left(\varepsilon_{R}\right) \approx \frac{1}{\sqrt{2 \pi\left\langle\left\langle\mathcal{E}_{R}^{2}\right\rangle\right\rangle}} \exp \left\{-\frac{\bar{\varepsilon}_{R}^{2}}{2\left\langle\left\langle\mathcal{E}_{R}^{2}\right\rangle\right\rangle}\right\}, \quad \bar{\varepsilon}_{R} \equiv \varepsilon_{R}-\left\langle\mathcal{E}_{R}\right\rangle,
$$

where $\left\langle\left\langle\mathcal{E}_{R}^{2}\right\rangle\right\rangle=\left\langle\mathcal{E}_{R}^{2}\right\rangle+\mathcal{O}\left(\alpha_{s}^{2}\right)$, with the first term given by (24).

At order $\lambda^{2}$, we may also begin to take into account the effects of the correlations between hemispheres, Eq. (37), in the shape function (8). Using the integral representation for the deltafunction entering (8), and applying the expansion (39) we get, for $\varepsilon_{R, L} \sim \Lambda$,

$$
f\left(\varepsilon_{R}, \varepsilon_{L}\right) \approx \int_{-\infty}^{\infty} \frac{d \lambda_{L}}{2 \pi} \frac{d \lambda_{R}}{2 \pi} \mathrm{e}^{i\left(\lambda_{L} \varepsilon_{L}+\lambda_{R} \varepsilon_{R}\right)} \exp \left\{-i\left\langle\left\langle\lambda_{L} \mathcal{E}_{L}+\lambda_{R} \mathcal{E}_{R}\right\rangle\right\rangle-\frac{1}{2}\left\langle\left\langle\left(\lambda_{L} \mathcal{E}_{L}+\lambda_{R} \mathcal{E}_{R}\right)^{2}\right\rangle\right\rangle\right\},
$$

where we have neglected contributions of higher cumulants. In this region, we find the approximate behavior

$$
f\left(\varepsilon_{R}, \varepsilon_{L}\right) \approx \frac{1}{2 \pi} \frac{1}{\sqrt{\mathcal{D}}} \exp \left\{-\frac{1}{2 \mathcal{D}}\left(\left\langle\left\langle\mathcal{E}_{L}^{2}\right\rangle\right\rangle \bar{\varepsilon}_{R}^{2}+\left\langle\left\langle\mathcal{E}_{R}^{2}\right\rangle\right\rangle \bar{\varepsilon}_{L}^{2}-2\left\langle\left\langle\mathcal{E}_{R} \mathcal{E}_{L}\right\rangle\right\rangle \bar{\varepsilon}_{R} \bar{\varepsilon}_{L}\right)\right\}
$$

Here the dispersion is defined as

$$
\mathcal{D}=\left\langle\left\langle\mathcal{E}_{L}^{2}\right\rangle\right\rangle\left\langle\left\langle\mathcal{E}_{R}^{2}\right\rangle\right\rangle-\left\langle\left\langle\mathcal{E}_{R} \mathcal{E}_{L}\right\rangle\right\rangle^{2}
$$

and clearly $\left\langle\left\langle\mathcal{E}_{R}^{2}\right\rangle\right\rangle=\left\langle\left\langle\mathcal{E}_{L}^{2}\right\rangle\right\rangle$. For small $\varepsilon_{R, L}$, the correlator $\left\langle\left\langle\mathcal{E}_{R} \mathcal{E}_{L}\right\rangle\right\rangle$ does not affect the asymptotic behavior of the shape function. Using (42) one finds $f\left(\varepsilon_{R}, \varepsilon_{L}\right) \sim f\left(\varepsilon_{R}\right) f\left(\varepsilon_{L}\right) \sim\left(\varepsilon_{R} \varepsilon_{L}\right)^{a_{\mathrm{NP}}-1}$ as $\varepsilon_{R, L} \rightarrow 0$. Together, these results imply, almost uniquely, the parameterization, Eq. (13) for the double-hemisphere shape function $f\left(\varepsilon_{R}, \varepsilon_{L}\right)$, as a minimal form, neglecting higher cumulants. The parameter $a$ of (13) has the interpretation of the particle multiplicity per unit rapidity radiated by the boost-invariant sources, while $b$ measures the extent to which radiation in one hemisphere, produced perturbatively or otherwise, "spills over" into the adjoining hemisphere, increasing correlations between $\varepsilon_{R}$ and $\varepsilon_{L}$.

The Gaussian fall-off of (13), of course, need not extend to arbitrarily large values of $\varepsilon_{L, R}$. For example, the large- $\varepsilon_{R, L}$ behavior that follows from the one-gluon approximation Eq. (40) is actually $\sim \exp \{-(\varepsilon / \Lambda) \ln (\varepsilon / \Lambda)\}$, which decreases somewhat less rapidly than the Gaussian in Eq. (13), although faster than an exponential. From a phenomenological point of view, however, this difference should have a modest effect on convolutions like Eq. (10) for physical cross sections.

To estimate the effect produced on the shape function (46) by nonzero correlations between the right and left hemispheres, we examine two extreme cases, $\left\langle\left\langle\mathcal{E}_{R} \mathcal{E}_{L}\right\rangle\right\rangle=\left\langle\left\langle\mathcal{E}_{L}^{2}\right\rangle\right\rangle$ and $\left\langle\left\langle\mathcal{E}_{R} \mathcal{E}_{L}\right\rangle\right\rangle=$ $-\left\langle\left\langle\mathcal{E}_{L}^{2}\right\rangle\right\rangle$, corresponding to maximal and minimal value of the correlator, respectively. Since in both cases the dispersion (47) vanishes, the shape function is reduced to $\delta\left(\varepsilon_{R}-\varepsilon_{L}\right)$ and $\delta\left(\varepsilon_{R}+\varepsilon_{L}-2\left\langle\mathcal{E}_{R}\right\rangle\right)$, 
correspondingly, indicating that the energy flows into the two hemispheres are strongly correlated with each other. For $\left\langle\left\langle\mathcal{E}_{R} \mathcal{E}_{L}\right\rangle\right\rangle>0$ this correlation is positive - as $\varepsilon_{R}$ increases, the same is true for $\varepsilon_{L}$. For $\left\langle\left\langle\mathcal{E}_{R} \mathcal{E}_{L}\right\rangle\right\rangle<0$ the situation is opposite - the energy flows into two hemispheres are anti-correlated with each other.

It follows from our calculation, Eq. (37), that the correlations between two hemispheres are positive. This property is in agreement with phenomenological fits of a Gaussian-type ansatz to experimental data [6] on event shapes. It is also in accord with the dispersive approach [18].

6. Conclusions. The event shape functions acquire an interpretation of energy flow distributions through hemispheres at infinities separated by the plane orthogonal to the jet axis. We have used perturbative QCD as a tool in our analysis of these functions, supplemented by conventional assumptions on the correspondence between perturbative and nonperturbative behavior [3]. While the resulting rapidity dependence is realistic, the transverse momentum dependence is governed by nonperturbative physics. The resumation of multi-energy correlators, in a one-gluon approximation, results in a power fall-off as $\varepsilon_{R, L} \rightarrow 0$. The sign of the correlation between the left and right hemispheres is more general than its perturbative approximation, and is a manifestation of the tendency of radiation into one hemisphere to spread into the other. As such, it is sensitive to the hadronization dynamics of soft radiation at wide angles from the jets. The functional form of the shape functions resulting from our approach is in the agreement with recent phenomenological analyses of differential event shape distributions [6, 7]. We expect to extend our results to all orders in the underlying perturbative expansion.

\section{Acknowledgements}

We are most grateful to Yu. Dokshitzer and A. Kaidalov for interesting discussions. A.B. is grateful to D. Schiff for the hospitality extended to him at the Laboratoire de Physique Théorique in Orsay. G.S. would like to thank Brookhaven National Laboratory for its hospitality. The work of A.B. and G.S. was supported in part by the National Science Foundation, grant PHY9722101. The work of G.K. was supported in part by the EU network 'Training and Mobility of Researchers', contract FMRX-CT98-0194.

\section{References}

[1] G. Sterman, S. Weinberg, Phys. Rev. Lett. 39 (1977) 1436.

[2] O. Biebel, Phys. Rept. 340 (2001) 165. 
[3] B.R. Webber, Phys. Lett. B 339 (1994) 148;

G.P. Korchemsky, G. Sterman, Nucl. Phys. B 437 (1995) 415; Universality of infrared renormalons in hadronic cross sections, in Proc. of the XXX Renconters de Moriond: QCD and High Energy Hadronic Interactions, ed. J. Trân Thahn Vân, Editions Frontiers, (Paris, 1995), p. 383; hep-ph/9505391;

Yu.L. Dokshitzer, B.R. Webber, Phys. Lett. B 352 (1995) 451;

R. Akhoury, V.I. Zakharov, Nucl. Phys. B 465 (1996) 295.

[4] G.P. Korchemsky, Shape functions and power corrections to the event shapes, in Proc. of the XXXIII Renconters de Moriond: QCD and High Energy Hadronic Interactions, ed. J. Trân Thahn Vân, Editions Frontiers, (Paris, 1999), p. 489; hep-ph/9806537.

[5] G.P. Korchemsky, G. Sterman, Nucl. Phys. B 555 (1999) 335.

[6] G.P. Korchemsky, S. Tafat, JHEP 0010 (2000) 010.

[7] E. Gardi, J. Rathsman, Renormalon resummation and exponentiation of soft and collinear gluon radiation in the thrust distribution, hep-ph/0103217.

[8] F.R. Ore, Jr., G. Sterman, Nucl. Phys. B 165 (1980) 93 (1980).

[9] N.A. Sveshnikov, F.V. Tkachev, Phys. Lett. B 382 (1996) 403;

P.S. Cherzor, N.A. Sveshnikov, Jet observables and energy momentum tensor, in Proc. of the 12th Inter. Workshop HEPQFT'97, ed. B.B. Levtchenko, p. 402; hep-ph/9710349;

M. Testa, JHEP 9809 (1998) 006.

[10] G.P. Korchemsky, G. Oderda, G. Sterman, Power corrections and nonlocal operators, in Proc. of 5th Inter. Workshop on Deep-Inelastic Scattering and QCD, eds. J. Repond, D. Krakauer, AIP Conf. Proc. No. 407, (New York, 1997), p. 988; hep-ph/9708346.

[11] S. Catani, L. Trentadue, G. Turnock, B.R. Webber, Nucl. Phys. B 407 (1993) 3.

[12] C.L. Basham, L.S. Brown, S.D. Ellis, S.T. Love, Phys. Rev. D 19 (1979) 2018.

[13] B.R. Webber, Hadronization, In Proc. of the Summer School on Hadronic Aspects of Collider Physics, (Zuoz, 1994), p. 49; hep-ph/9411384.

[14] G. Sterman, Infrared divergences in perturbative QCD, in Proc. of Tallahassee Conference on Perturbative Quantum Chromodynamics, eds. D.W. Duke, J.F. Owens, AIP Conf. Proc. No. 74, (New York, 1981), p. 22;

J.G. Gatheral, Phys. Lett. B 133 (1983) 90. 
[15] A.V. Belitsky, Phys. Lett. B 442 (1998) 307.

[16] N.G. van Kampen, Physica 74 (1974) 239; Phys. Rept. C 24 (1976) 171.

[17] A.H. Mueller, Phys. Rev. D 4 (1971) 150;

K. Fialkowski, Correlations and fluctuations: Introduction, hep-ph/0101016.

[18] Yu.L. Dokshitzer, G. Marchesini, B.R. Webber, JHEP 9907 (1999) 012. 